\title{
Crumbs from the Rich Man's Iable
}

At least so far as molecular biology is concerned the New Year has got off to a bright start. Much sweat has flowed under the bridge since transfer RNA was first crystallized. The incandescence of the early optimism gradually gave place to plaintive tales of unstable crystals, nonisomorphous replacements, twinning, and all the other calamities that crystallographers are heir to. Yet here suddenly come Kim and his eight colleagues from Rich's laboratory at the Massachusetts Institute of Technology, tripping down the primrose path to atomic resolution.

Success was evidently contingent, as so often in the early days of protein crystallography, on the witchcraft of good isomorphous replacement. In an article in the December issue of the Proceedings of the National Academy of Sciences $(69,3746 ; 1972) \mathrm{Kim}$ et al. refer to the preparation of three heavy-atom derivatives, one containing three platinum atoms, the second two samarium and the third an osmium. It was the last that proved crucial; it involved first the preparation of a nucleotide osmic ester, from ATP and a bipyridine osmate complex. When this was diffused into the tRNA crystal it reacted stoichiometrically at a single site, the $3^{\prime}$ terminus, where sugar cis hydroxyl groups are available for complex formation. In this article a resolution of $5.5 \AA$ was described-barely sufficient to reveal the outlines of the molecule, which was surmised only to be elongated with here and there some constrictions. The molecules are set well apart in the lattice, and in between are solventpermeated channels. Here and there what seemed to be the phosphodiester chain could be made out, and a loop appeared to run round the outside of a part of the molecule.

Only another two months sufficed to give these shadows substance in a $4 \AA$ model (Kim et al., Science, 179, 285 ; 1973). Here concentrations of electron density corresponding to the individual nucleotides can be discerned. The molecule resolves itself into a rough $\mathrm{L}$-shape. The course of the backbone is for the most part easily traced by moving from one maximum to another the right distance away. Where the assignment is equivocal, it can be resolved by reference to the base-pairing scheme imposed by the cloverleaf model. Complete consistency between the cloverleaf scheme and the conformation in the crystal is in fact indicated, though the tertiary structure is far from cruciform. The authors are collecting data at $3 \AA$, which will allow them to define the structure uniquely without regard to external evidence.

It already seems, however, that there can be little doubt about the correctness of the model. The most striking feature is that there are only two lengths of double helix, each of about one turn. This means that the base-paired stems are collinear in pairs. The duplex formed by the two terminal segments of the chain is continuous with the stem of the pseudouridine loop, and is connected in the middle by way of two single-stranded segments (one of them the "extra loop") to the second double helix, made up of the base-paired stems of the dihydrouracil and the anticodon loops, the two helices lying more or less at right angles to one another. The variable length of the "extra loop", which has anything from four to twenty-one residues in the tRNA species of known sequence, can be readily accommodated, because it lies on the outside, linking the two arms of the $\mathrm{L}$ at their centres. Four residues in fact is the minimum length that will span the distance. The acceptor end, the 2' hydroxyl $\mathrm{CpCpA}$, projects outwards at an angle, and may be free to take up different orientations at will.

The implications of the model will have to be digested at leisure, but the authors have already noted that it accounts readily enough for various features of the chemistry, such as photodimer formation between the thiouracil residue at position 8 and cytosine at position 13 . Three-dimensional models based on inferences from chemical and physical properties have, of course, dropped into the literature and vanished as rain upon the desert's dusty face; none, one may confidently predict, will survive this model. Only the base-pairing-Holley's original cloverleaf-seems unassailable. Moreover, a comparison of the X-ray data with earlier and more rudimentary results from the same laboratory on another species, formylmethionine tRNA, indicates that the gross structures must be very similar. This encourages the hope that all tRNAs will fit into the same mould structurally, just as their base-pairing schemes conform to the cloverleaf. The $3 \AA$ model, which should yield, among other bonuses, the long-awaited proof that the base-pairing is of the Watson-Crick type (or not as the case may be), as well as a wealth of miscellaneous structural information, to illuminate accumulated experimental data, will be eagerly awaited.-From our Molecular Biology Correspondent.

\section{Human Papova Viruses}

To the numerous laboratories throughout the world that are preoccupied with cancer research the recent discovery of latent human papova viruses must come like manna. With a minimum of effort such laboratories can now submit research proposals and grant applications that surely stand a good chance of being accepted and funded. All they have to do is to propose to repeat, using human papova virus(es), the elegant and important experiments that have been carried out with simian virus 40 during the past several years. One thing seems incontestable; this new human virus is the human counterpart of SV40, a latent monkey virus that induces tumours in rodents if not in monkeys and transforms rodent cells growing in culture.

The human papova virus (whether there is just one species or several related species is not yet known) first came to light in 1971 when Gardner and her colleagues detected it in the urine of a person who had undergone kidney transplant surgery and was being treated with immunosuppressives. Subsequently the same virus or a very closely related virus was detected by Weiner and his colleagues in biopsies of brain tissue from patients with progressive multifocal leucoencephalopathy and 